\title{
Optimal transmission switching to eliminate voltage violations during light-load periods using decomposition approach
}

\author{
Boshi ZHAO ${ }^{1}$, Zechun HU $^{1}{ }^{\mathbb{D}}$, Qian ZHOU $^{2}$, Hongcai ZHANG $^{1}$, \\ Yonghua SONG ${ }^{1,3}$
}

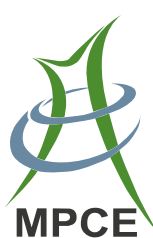

\begin{abstract}
With the rapid load increase in some countries such as China, power grids are becoming more strongly interconnected, and the differences between peak and valley loads are also increasing. As a result, some bulk power systems are facing high voltage limit violations during light-load periods. This paper proposes to utilize transmission switching (TS) to eliminate voltage violations. The TS problem is formed as a mixed-integer nonlinear program (MINLP) with AC power flow constraints and binary variables. The proposed MINLP problem is non-deterministic polynomial hard. To efficiently solve the problem, a decomposition approach is developed. This approach decomposes the original problem into a mixedinteger linear programming master problem and an $\mathrm{AC}$
\end{abstract}

CrossCheck date: 17 April 2018

Received: 14 March 2017/Accepted: 17 April 2018/Published online: 17 July 2018

(C) The Author(s) 2018

$\triangle$ Zechun HU

zechhu@tsinghua.edu.cn

Boshi ZHAO

zbsisaman@163.com

Qian ZHOU

xjtu@163.com

Hongcai ZHANG

zhang-hc09@mails.tsinghua.edu.cn

Yonghua SONG

yhsong@mail.tsinghua.edu.cn

1 Department of Electrical Engineering, Tsinghua University, Beijing 100084, China

2 State Grid Jiangsu Electric Power Research Institute, Nanjing 211103, China

3 Department of Electrical and Computer Engineering, University of Macau, Macau, China optimal power flow slave problem that is used to check the AC feasibility. Prevention of islanding is also taken into consideration to ensure the feasibility of the TS results. The modified IEEE 39-bus and IEEE 57-bus test systems are used to demonstrate the applicability and effectiveness of the proposed method.

Keywords Transmission switching, Voltage violation, Optimal power flow, Decomposition approach, Mixedinteger programming

\section{Introduction}

As the transmission network is expanded and reinforced with load increase and more power generation integrations, it is difficult to maintain the nodal voltage magnitude in allowable ranges under all operational conditions. For example, it is a newborn problem that transmission systems in China are facing serious high voltage limit violations during light-load periods, such as festivals and holidays. The massive gap between the peak and valley load power, the widespread use of long-distance and large-section transmission lines, and inadequate inductive reactive power reserves are the three key factors that result in the voltage violation problem. Even if synchronous generators operate in the leading-phase state and shunt reactors are all switched on, the capacitive reactive power is still excessive, because of which the voltage magnitudes violate the upper limits for some buses.

Because switching transmission lines can change the network topology and consequently alter the distribution of power flows, switching out some transmission lines can be an alternative voltage control method. Switching out transmission lines can physically remove the equivalent 
shunt capacitors of the lines. In addition, switching out lines will lead to the power flow increases of other lines indirectly, and thereby increase the consumption of reactive power. Some power system operators in different regions of China have chosen to switch out some transmission lines to relieve the voltage violations during spring festivals in recent years. However, the system operators usually make the transmission line switching decisions on the basis of their experience. The choices of the transmission lines to be switched out and the coordination with other voltage control measures are actually a type of optimal transmission switching (TS) problem. An appropriate mathematical model and method for the solution should be developed to provide an optimal TS scheme for the system operators.

For more flexible and smarter power systems, TS is a relatively advanced and valuable method for researchers and operators, which contributes to better operational objectives by reconfiguring the topologies of power systems. TS was developed to solve different types of problems such as unit commitment involving transmission congestion management [1-6]. In [1, 2], contingencies are considered to examine the reliability of the topologies after TS. In [3] voltage security is considered in economic dispatch problem. Islanding prevention and node-breaker modeling are respectively considered in [4, 5]. In [6], the performances of TS in the Central Western European market prove significant cost savings. Furthermore, TS is also adopted as a measure of contingency precaution and recovery for security enhancement of system operation [7-9].

However, the voltage control and reactive power optimization problems should be based on the AC power flow models. Therefore, with the binary variables corresponding to the on/off states of transmission lines, this type of TS problem can be formulated as mixed-integer nonconvex and nonlinear problems, which are quite difficult to solve. Thus, computational complexity is one of the main challenges [10]. Researchers have made many attempts to find effective methods to solve AC optimal TS (ACOTS) problems. In [11-15], linearization methods are used to deal with loss reduction or voltage violation problems. The best switching actions are determined iteratively using sensitivities or by formulating a linear programming model. Reference [14] adopts the fast decoupled power flow and voltage distribution factors to find the best lines and bus-bars of transformer substations to switch in order to relieve overloads and voltage violations. In [15], a linearization method suitable for the light-load state is proposed to analyze the reactive power and voltage magnitude.

In [16-18], the ACOTS problems are formulated as semi-definite programming (SDP) and second-order cone programming (SOCP) models by $\mathrm{AC}$ power flow relaxation. However, these models cannot ensure AC power flow feasibility and only provide lower bounds for the original problems [17], which is not adequate for the practical operation problem. In [18], a two-level iterative approach is developed to check the feasibility of the SOCP results. The additional constraints of switched-out line combinations are formulated by the lower-level problem when the AC feasibility check fails.

Heuristic algorithms are also used in solving the ACOTS problems [19, 20]. In [19], the accuracies of ACOTS and DC optimal TS (DCOTS) are compared using heuristic algorithms. The results show that DCOTS model may be inaccurate and performs poorly in several cases. In [20], the DCOTS approximation model, the SDP and SOCP relaxation methods, and the original AC model solved by heuristic algorithms are compared. The results demonstrate that the DC power flow model is not reliable for obtaining optimal line switching results, while the heuristic algorithms can be valuable for finding AC feasible solutions, and the relaxation method is instrumental in guaranteeing the optimization objective. Benders decomposition approach divides the original problem into a master and a slave problem to reduce the constraints of the original problem [21-23]. In [3], a Benders decomposition algorithm is also used to solve the TS problem and the slave problem is used to check $\mathrm{AC}$ feasibility and $\mathrm{N}-1$ security criteria.

In this paper, we study the optimal TS problem with AC power flow to eliminate the voltage violation during the light-load periods. The voltage security constraints and AC feasibility are guaranteed in the model, which avoids the aforementioned shortcomings of DCOTS [11-15]. A decomposition approach is used to decompose the original problem into a mixed-integer linear programming master problem and an AC optimal power flow (ACOPF) slave problem. Prevention of islanding is also taken into consideration in this method. The main contributions of the paper are as follows:

1) The model solving the voltage violation problems by optimal TS is built in ACOTS formulation.

2) A decomposition method is employed to decompose the ACOTS model into solvable master and slave problems, which can be solved efficiently by iteration.

3) The ACOPF slave problem is solved by interior point method and the Jacobian and Hessian matrices are derived considering the change of the power network topology, which can also be used in other optimal TS problems.

The rest of the paper is organized as follows. Section 2 introduces the formulation of the ACOTS model and the linearization procedure for the model. Section 3 describes 
the solution method for the ACOTS model based on a decomposition approach. Section 4 presents simulation results using the proposed method on two test systems. Section 5 concludes the paper.

\section{ACOTS formulation and linearization for light- load state}

\subsection{Formulation of ACOTS model}

In this section, the ACOTS problem is formulated as a mixed-integer nonlinear program (MINLP) problem:

$$
\begin{aligned}
& \min \left(\sum_{i=1}^{n_{b}} \beta_{i} v_{i}^{\text {low }}+\sum_{i=1}^{n_{b}} \chi_{i} v_{i}^{\text {up }}+\delta \sum_{i=1}^{n_{g}} C_{i} P_{G i}\right) \\
& \text { s.t. } \\
& V_{i}^{\text {min }}-v_{i}^{\text {low }} \leq V_{i} \leq V_{i}^{\text {max }}+v_{i}^{\text {up }} \\
& \left\{\begin{array}{l}
v_{i}^{\text {low }} \geq 0 \\
v_{i}^{\text {up }} \geq 0
\end{array}\right. \\
& P_{G i}-P_{D i}=V_{i} \sum_{j \in i} V_{j}\left(z_{i j} G_{i j} \cos \theta_{i j}+z_{i j} B_{i j} \sin \theta_{i j}\right) \\
& P_{G i}^{\min } \leq P_{G i} \leq P_{G i}^{\max } \\
& Q_{G i}-Q_{D i}=V_{i} \sum_{j \in i j \neq i} V_{j}\left(z_{i j} G_{i j} \sin \theta_{i j}-z_{i j} B_{i j} \cos \theta_{i j}\right) \\
& Q_{G i}^{\min } \leq Q_{G i} \leq Q_{G i}^{\max }\left[B_{i i}-B_{i j}^{C}\left(1-z_{i j}\right) / 2\right] \\
& S_{i j}=\sqrt{P_{i j}^{2}+Q_{i j}^{2}} \\
& z_{i j} S_{i j}^{\min } \leq S_{i j} \leq z_{i j} S_{i j}^{\max } \\
& \sum_{(i, j) \in L}\left(1-z_{i j}\right) \leq N_{S}
\end{aligned}
$$

where $n_{b}, n_{g}$ are the total number of buses and generators, respectively; $\delta$ is the weighting factor of generation costs; $\beta_{i}$ and $\chi_{i}$ are weighting factors; $V_{i}, V_{j}$ are voltage magnitudes at Bus $i$ and Bus $j ; v_{i}^{\text {low }}, v_{i}^{\text {up }}$ are the lower and upper slack quantities of voltage magnitude limit; $P_{G i}, Q_{G i}$ are active and reactive power generation at Bus $i ; P_{D i}, Q_{D i}$ are active and reactive power demands at Bus $i ; \theta_{i j}$ is voltage phase angle difference between Bus $i$ and Bus $j ; z_{i j}$ is the binary variable representing the switching state of Line $(i$, j) (0: open, 1: closed); $P_{i j}, Q_{i j}$ and $S_{i j}$ are active power, reactive power and apparent power flow of Line $(i, j) ; G_{i j}$, $B_{i j}$ are elements of the conductance matrix and susceptance matrix; $B_{i j}^{C}$ is susceptance of charging capacitor of Line $(i$, $j)$; superscripts min and max represent minimum and maximum values for corresponding variables; $L$ is the set of transmission lines; $N_{S}$ is the maximum number of switched-out lines.

The objective is to minimize the sum of the slack quantities of the voltage magnitude violations and the generation costs in the system. The slack quantities reflect the extent of the nodal voltage violation, while the corresponding weighting factors $\beta_{i}$ and $\chi_{i}$ can be set as same, or determined by considering other factors.

The values of the slack quantities of the voltage magnitude limit, which are defined in (2), are nonnegative as shown in (3). Equations (4) and (6) are the AC power flow constraints with the binary variables of the line switching state. Equations (5) and (7) are the constraints of the lower and upper limits for power generation. Equation (9) denotes the power flow limits on the lines. Equation (10) is the limit of the total number of lines that can be switched out.

This optimization problem determines the lines to be switched out and the dispatch of generators' reactive and active power for the purpose of relieving voltage violations. Actually, different load levels are not considered in this model. The TS optimal results are determined on the day ahead based on the average load level of the next day with the solutions of the model proposed.

\subsection{ACOTS linearization for light-load state}

The aforementioned ACOTS model is an MINLP problem. Available solvers for MINLP problems are not effective for this ACOTS model. A linearization method proposed in [15] is adopted in this part, which is useful for the light-load operational state.

For the large-scale transmission system operating in a light-load condition, the constraints involving the active power, i.e., constraints (4), (6), (8), and (9), can be ignored for the voltage magnitude optimization, which are considered in the slave problem.

The reactive power on a line can be quantified using (11) and (12). The line is modeled in the form of $\pi$ equivalence. Equation (11) represents the reactive power flow on the connected Line $(i, j)$. Equation (12) is the charging reactive power of the line to Bus $i$.

$Q_{i j}=V_{i}^{2} B_{i j}+V_{i} V_{j} G_{i j} \sin \theta_{i j}-V_{i} V_{j} B_{i j} \cos \theta_{i j}$

$Q_{i j-i}^{C}=\frac{B_{i j}^{C}}{2} V_{i}^{2}$

Because the active power flow is not heavy during the light-load period, the $\cos \theta_{i j}$ term in (11) can be approximated as 1.0. Similarly, $\sin \theta_{i j}$ and $G_{i j}$ are both small. Thus, the second term of the right side of (11) can be omitted. 
Because the voltage magnitude is close to 1.0 p.u., the Taylor expansion can be used to approximate the quadratic term as follows:

$V_{i}^{2}=2 V_{i}-1$

$V_{i} V_{j}=V_{i}+V_{j}-1$

Thus, (11) and (12) can be derived as follows:

$Q_{i j}=\left(V_{i}-V_{j}\right) B_{i j} z_{i j}$

$Q_{i j-i}^{C}=\frac{B_{i j}^{C}}{2}\left(2 V_{i}-1\right) z_{i j}$

Actually, (15) and (16) are quadratic constraints, because $V_{i}$ and $z_{i j}$ are both variables. Therefore, $W_{k}$ is introduced to replace the product of $V_{i}$ and $z_{i j}$, which is defined by (17) and (18). Add constraints (17) and (18) into the model, and then the quadratic terms can be eliminated.

$V_{i}^{\min } z_{i j} \leq W_{k} \leq V_{i}^{\max } z_{i j}$

$0 \leq V_{i}-W_{k} \leq\left(1-z_{i j}\right) V_{i}^{\max }$

Now, the ACOTS problem can be linearized as a mixedinteger linear programming problem, which is formulated in detail in Sect. 3 as the master problem for the decomposition approach.

\section{Solution method based on a decomposition approach}

\subsection{Model based on a decomposition approach}

During light-load periods, the ACOTS problem can be divided into a traditional active power DCOPF model and a reactive power OPF model.

The main purpose of this paper is to deal with voltage security problem. Therefore, we get the economic dispatch result first with the active power DCOPF model without switching lines. The result is used to determine the active power outputs used in the mathematic formulation of this work. In practice, the active power outputs can be determined by operation schedule.

As for the reactive power OPF model, TS is used to eliminate voltage violations. Hence, the model can be formulated as a mixed-integer linear program with the linearization method described in Sect. 2, which can be solved easily using off-the-shelf software such as CPLEX Solver [24].

However, according to our simulation tests, the results obtained with the linearization model may be infeasible for $\mathrm{AC}$ power flow equations, and the reactive power error between the $\mathrm{AC}$ and the linearized power flow models cannot be ignored in many cases. Therefore, an ACOPF slave problem is proposed in this paper in order to check the AC feasibility of the results of linearized model and obtain a local optimal solution based on the TS results.

A decomposition approach used in this paper is a suitable method to solve the whole problem, which is similar to but different from standard Benders decomposition approach. The differences include that the master problem is a simplified form of the slave problem and the aims of the master and slave problems are different. The additional constraints are formulated by the slave problem just as the Benders decomposition approach. Thus, the constraints are also called "Benders cuts" in this paper, which are useful to determine the solving direction and guarantee the solving efficiency of the problem with large numbers of integer variables. They are formed and added to the linearized problem if the result cannot pass the AC feasibility check. Similar idea is used in [25]. The applicability and robust features of the decomposition approach are discussed in Sect. 3.2.

\section{1) Master problem}

The master problem of the decomposition is the linearized models of the ACOTS problem. The traditional active power DCOPF model is omitted in this paper. The objective function of reactive power OPF model is defined as (19), whose constraints contain (2), (3), (7), (10), (15), (16), (20)-(22).

$$
\begin{aligned}
& \min \left(\sum_{i=1}^{n_{b}} \beta_{i} v_{i}^{\mathrm{low}}+\sum_{i=1}^{n_{b}} \chi_{i} v_{i}^{\mathrm{up}}\right) \\
& Q_{C i, n}=\left[\left(2 V_{i}-1\right) b_{C i}\right] z_{C i, n} \\
& Q_{L i, n}=-\left[\left(2 V_{i}-1\right) b_{L i}\right] z_{L i, n} \\
& Q_{G i}+\sum_{i} Q_{i j-i}^{C}+\sum_{n=1}^{n_{C i}} Q_{C i, n}+\sum_{n=1}^{n_{L i}} Q_{L i, n}-\left(Q_{D i}+\sum_{i} Q_{i j}\right)=0
\end{aligned}
$$

where $b_{C i}, b_{L i}$ are admittance values of the capacitors and inductors of Bus $i ; z_{C i, n}, z_{L i, n}$ are the binary variables representing the switching state of the shunt capacitors and inductors (0: open, 1: closed); $Q_{C i, n}, Q_{L i, n}$ are reactive power of the shunt capacitors and inductors; $n_{C i}$ and $n_{L i}$ are the total number of the shunt capacitors and inductors.

Equations (20) and (21) represent the equality constraints of the reactive power of the shunt capacitors and inductors, in which the binary variables representing their switching state can also be optimized in this problem. Equation (22) represents the constraints of the reactive power balance on Bus $i$.

\section{2) Slave problem}

The slave problem is used to check the feasibility of the solution given by the master problem using the AC power flow model. The switching states of the candidate lines are 
set to the values determined in the master problem. The objective function of the slave problem is formulated as (23), whose constraints contain (7)-(9) and (24)-(31).

$$
\begin{aligned}
& \min \eta=\sum_{i=1}^{n_{\mathrm{PQ}}}\left(v_{\mathrm{PQ}, i}^{\mathrm{up}}+v_{\mathrm{PQ}, i}^{\mathrm{low}}\right) \\
& V_{\mathrm{PV}, i}=\hat{V}_{\mathrm{PV}, i} \leftrightarrow \lambda_{V_{\mathrm{PV}, i}} \\
& z_{i j}=\hat{z}_{i j} \leftrightarrow \lambda_{z_{i j}} \\
& \max \left(V_{\mathrm{PV}, i}(1-\gamma), V_{\mathrm{PV}, i}^{\min }\right) \leq \bar{V}_{\mathrm{PV}, i} \leq \min \left(V_{\mathrm{PV}, i}(1+\gamma), V_{\mathrm{PV}, i}^{\max }\right) \\
& V_{\mathrm{PQ}, i}^{\min }-v_{\mathrm{PQ}, i}^{\text {low }} \leq \bar{V}_{\mathrm{PQ}, i} \leq V_{\mathrm{PQ}, i}^{\mathrm{max}}+v_{\mathrm{PQ}, i}^{\mathrm{up}} \\
& \left\{\begin{array}{l}
v_{\mathrm{PQ}, i}^{\text {low }} \geq 0 \\
v_{\mathrm{PQ}, i}^{\mathrm{up}} \geq 0
\end{array}\right. \\
& P_{G i}-P_{D i}=\bar{V}_{i} \sum_{j \in i} \bar{V}_{j}\left(z_{i j} G_{i j} \cos \theta_{i j}+z_{i j} B_{i j} \sin \theta_{i j}\right) \\
& \max \left(\hat{P}_{G i}(1-\varepsilon), P_{G i}^{\min }\right) \leq P_{G i} \leq \min \left(\hat{P}_{G i}(1+\varepsilon), P_{G i}^{\max }\right) \\
& Q_{G i}-Q_{D i}=\bar{V}_{i} \sum_{j \in i, j \neq i} \bar{V}_{j}\left(z_{i j} G_{i j} \sin \theta_{i j}-z_{i j} B_{i j} \cos \theta_{i j}\right) \\
& -\bar{V}_{i}^{2}\left[B_{i i}-B_{i j}^{C}\left(1-z_{i j}\right) / 2\right]
\end{aligned}
$$

where $n_{\mathrm{PQ}}$ is the total number of the PQ buses; $\hat{V}_{\mathrm{PV}, i}$ and $\hat{z}_{i j}$ are the values of the voltage magnitudes of the PV buses and the switching states determined in the master problem, respectively; $\lambda_{V_{\mathrm{PV}, i}}, \lambda_{z_{i j}}$ are respectively the Lagrangian coefficients of the corresponding equality constraints; $\varepsilon$ is the factor of active power generation adjustment; $\gamma$ is the factor of voltage magnitude adjustment of PV buses from master problem to slave problem; $\bar{V}_{\mathrm{PV}, i}$ represents the voltage magnitude of the PV buses of the AC power flow constraints, which can be adjusted from $V_{\mathrm{PV}, i}$ by $\gamma ; \bar{V}_{\mathrm{PQ}, i}$ is the voltage magnitude of the PQ buses of the AC power flow equations; $\bar{V}_{i}$ and $\bar{V}_{j}$ are voltage magnitudes omitting the type of buses; $\hat{P}_{G i}$ is the value of the active power outputs of generators determined in the omitted active power DCOPF model.

The objective function (23) is the sum of the slack quantities of the voltage magnitude limits of the PQ buses. The objective function here reflects not only the extent of voltage violations but also the AC feasibility of the optimization results, so the weighting factors of the slack quantities are no longer considered. If the objective is smaller than a predefined positive number, the slave problem is considered to be feasible, the iterative procedure ends, and the TS solutions are obtained. In (24) and (25), $V_{\mathrm{PV}, i}$ and $z_{i j}$ are set equal to the value determined in the master problem. The Lagrangian coefficients of the equations are used in the Benders cuts formulation. Equation (26) gives the ranges of the voltage magnitude constraints of the PV buses. Equation (27) defines the slack quantities of the voltage magnitude limits of the PQ buses. Equation (30) limits the ranges of the active power generation that can be adjusted for voltage security. It is assumed that the active power outputs of the generators can be changed in a small range, around the economic dispatch results, defined by $\varepsilon$, which is a small positive number adjusted by the user. Equations (29) and (31) are the AC power flow constraints in the slave problem.

\section{3) Benders cut}

The Benders cut can be formed on the basis of the optimization results of the slave problem as follows:

$$
\eta+\sum_{(i, j) \in L} \lambda_{z_{i j}}\left(z_{i j}-z_{i j}^{k-1}\right)+\sum_{i=1}^{n_{\mathrm{PV}}} \lambda_{V_{\mathrm{PV}, i}}\left(V_{\mathrm{PV}, i}-V_{\mathrm{PV}, i}^{k-1}\right) \leq 0
$$

where $z_{i j}^{k-1}$ and $V_{\mathrm{PV}, i}^{k-1}$ represent the optimization results of the switching states and the voltage magnitudes of the PV buses at iteration $k-1$, respectively. The Benders cut is added to the master problem in each iteration $k$. The Lagrangian coefficients in (32) denote the influence of the change of $z_{i j}$ and $V_{\mathrm{PV}, i}$ on the objective function value of the slave problem, which gives the adjustment direction of the variables. Thus, the Benders cuts couple the master and the slave problems in order to remove the AC-infeasible region of the master problem until the optimization results satisfy all the required constraints.

4) Procedure of decomposition approach

The algorithm is processed as follows:

Step 1: Solve the master problem, and pass the switching states of candidate lines and voltage magnitudes of the PV buses to the slave problem.

Step 2: Solve the slave problem, and check the objective value. If it is smaller than the predefined small value, the problem is solved; otherwise, go to Step 3. If the slave problem cannot get feasible solutions, go to Step 4 .

Step 3: Form the new constraint cut on the basis of the optimization results of the slave problem and add it to the master problem as a new constraint. Go back to Step 1 .

Step 4: Unsolvable slave problem means that the switching decision is infeasible. Formulate the constraint (33) that the lines in the set cannot be switched out at the same time to the master problem, and go back to Step 1 .

$\sum_{m \in L_{k}} z_{m} \geq 1$ 
where $L_{k}$ is the switched-out line set determined by the master problem in the $k$ th iteration time.

\subsection{Applicability analysis of decomposition approach}

The above slave problem with AC power flow constraints is non-convex. It is possible that the global optimal solution is eliminated by Benders cuts. We can partially resolve the problem by finding multiple solutions [25]. After finding a feasible solution, the solving process can be executed again by excluding the TS results found in the preceding iterations. In this way, multiple solutions can be found and compared from different perspectives, such as the switched-out costs of different transmission lines. Even if the voltage violations cannot be eliminated thoroughly, the TS schemes and reactive power dispatch results can be obtained optimally, which are useful for real application.

\subsection{Derivation of matrix used in primal-dual interior point method}

The slave problem is a nonlinear programming model without integer variables, which can be effectively solved by the primal-dual interior point method [26, 27]. The MATPOWER toolbox, an open-source MATLAB-based package introduced in [28], is employed. In MATPOWER, the primal-dual interior point method is used to solve the ACOPF problem. Although the line switching states are determined in the master problem and fixed in the slave problem, they must be treated as variables, because the corresponding Lagrangian coefficients of constraints (24) and (25) should be calculated. To calculate the Lagrangian coefficients of the Benders cuts, the Jacobian matrix and Hessian matrix of all the variables including the binary variables of the line switching states need to be calculated in each iteration.

However, the binary variables of the line switching states are quite different from other variables in the structures of power flow equations. Therefore, the corresponding matrices should be derived for the OPF problem taking the topological change into consideration.

The AC power flow equality constraints can be defined as follows in the form of complex matrices, which are also presented in the MATPOWER OPF solver:

$\Delta \boldsymbol{S}=\operatorname{diag}(\boldsymbol{V})\left(\boldsymbol{Y} \boldsymbol{V}^{*}\right)^{*}-\boldsymbol{S}$

where $\boldsymbol{Y}$ is the $n_{b} \times n_{b}$ complex admittance matrix; $\boldsymbol{S}$ is the $n_{b} \times 1$ vector of nodal power injections; $\Delta S$ is the $n_{b} \times 1$ vector of mismatch of bus power injections; $\boldsymbol{V}$ is the $n_{b} \times 1$ vector of complex bus voltages; $\operatorname{diag}(\boldsymbol{V})$ is the $n_{b} \times n_{b}$ diagonal matrix whose diagonal elements are the elements of vector $\boldsymbol{V}$.

The switching of branches will change the admittance matrix, which can be denoted as follows:

$$
\begin{aligned}
\boldsymbol{X}=\boldsymbol{M}_{b l} & \operatorname{diag}(\boldsymbol{y}) \operatorname{diag}(\boldsymbol{z}) \boldsymbol{M}_{b l}^{\mathrm{T}} \\
& +\operatorname{diag}\left(\operatorname{abs}\left(\boldsymbol{M}_{b l}\right) \operatorname{diag}(\boldsymbol{z})\left(\boldsymbol{B}_{c} / 2\right)\right)
\end{aligned}
$$

where $\boldsymbol{y}$ is the $n_{l} \times 1$ vector of complex admittance of lines; $\boldsymbol{z}$ is the $n_{l} \times 1$ vector of switching states of lines; $\boldsymbol{M}_{b l}$ is the $n_{b} \times n_{l}$ bus-to-line incidence matrix, which reflects the relationship connecting buses and lines; $\operatorname{abs}\left(\boldsymbol{M}_{b l}\right)$ is the matrix with absolute value of $\boldsymbol{M}_{b l} ; \boldsymbol{B}_{c} / 2$ represents the elements in the vector of the charging susceptance divided by 2 .

Therefore, the AC power flow equality constraints with switching variables can be defined as follows:

$\Delta \boldsymbol{S}=\operatorname{diag}(\boldsymbol{V})\left(\boldsymbol{X} \boldsymbol{V}^{*}\right)^{*}-\boldsymbol{S}$

The main submatrices of the Jacobian matrix can be derived as follows:

$\frac{\partial \Delta \boldsymbol{S}}{\partial \boldsymbol{V}_{m}}=\operatorname{diag}(\boldsymbol{V})\left(\operatorname{diag}\left(\boldsymbol{I}^{*}\right)+\boldsymbol{X}^{*} \operatorname{diag}\left(\boldsymbol{V}^{*}\right)\right) \operatorname{diag}\left(\boldsymbol{V}_{m}\right)$

$\frac{\partial \Delta \boldsymbol{S}}{\partial \boldsymbol{\theta}}=\operatorname{jdiag}(\boldsymbol{V})\left(\operatorname{diag}\left(\boldsymbol{I}^{*}\right)-\boldsymbol{X}^{*} \operatorname{diag}\left(\boldsymbol{V}^{*}\right)\right)$

$\boldsymbol{I}=\boldsymbol{X V}$

where $\boldsymbol{I}$ is the $n_{b} \times 1$ vector of complex current injections; $\boldsymbol{V}_{m}$ is the $n_{b} \times 1$ vector of voltage magnitudes; $\partial \Delta \boldsymbol{S} / \partial \boldsymbol{V}_{m}$ is the $n_{b} \times n_{b}$ Jacobian submatrix comprising derivatives between the mismatches of the bus complex power injection and the voltage magnitudes; $\partial \Delta \boldsymbol{S} / \partial \boldsymbol{\theta}$ is the $n_{b} \times$ $n_{b}$ Jacobian submatrix comprising derivatives between the mismatches of the bus complex power injection and the voltage magnitudes phase angle.

$$
\begin{aligned}
\frac{\partial \Delta \boldsymbol{S}}{\partial z}= & \operatorname{diag}(\boldsymbol{V}) \boldsymbol{M}_{S z} \operatorname{diag}\left(\boldsymbol{y}^{*}\right) \\
& +\operatorname{diag}\left(\boldsymbol{V}_{m}^{2}\right) \operatorname{abs}\left(\boldsymbol{M}_{b l}\right) \operatorname{diag}\left(\boldsymbol{B}_{c}^{*} / 2\right) \\
\boldsymbol{M}_{S z}= & {\left[\boldsymbol{M}_{b l}(:, 1) \boldsymbol{M}_{b l}^{\mathrm{T}}(:, 1) \boldsymbol{V}^{*} \ldots \boldsymbol{M}_{b l}\left(:, n_{l}\right) \boldsymbol{M}_{b l}^{\mathrm{T}}\left(:, n_{l}\right) \boldsymbol{V}^{*}\right] }
\end{aligned}
$$

where $\partial \Delta \boldsymbol{S} / \partial \boldsymbol{z}$ is the $n_{b} \times n_{l}$ Jacobian submatrix comprising the derivatives between the mismatches of the bus complex power injection and the switching variables; $M_{S z}$ is the $n_{b} \times n_{l}$ matrix, the $i$ th column of which is the $n_{b} \times 1$ vector $\boldsymbol{M}_{b l}(:, i) \boldsymbol{M}_{b l}^{\mathrm{T}}(:, i) \boldsymbol{V}^{*}$.

In addition, the Hessian matrix, which is composed of the second derivative of $\Delta S^{\mathrm{T}} \lambda$ with respect to its variables, needs to be derived, where $\lambda$ is the $n_{b} \times 1$ vector of Lagrangian coefficients. The Hessian submatrices with the switching variables are derived as follows: 
$\frac{\partial^{2}\left(\Delta \boldsymbol{S}^{\mathrm{T}} \boldsymbol{\lambda}\right)}{\partial \boldsymbol{z}^{2}}=\mathbf{0}$

$$
\begin{aligned}
\frac{\partial^{2}\left(\Delta \boldsymbol{S}^{\mathrm{T}} \boldsymbol{\lambda}\right)}{\partial \boldsymbol{z} \partial \boldsymbol{V}_{m}}= & \left.2 \operatorname{diag}\left(\boldsymbol{y}^{*}\right) \operatorname{real}\left(\boldsymbol{M}_{S z \nu} \operatorname{diag}(\boldsymbol{\lambda}) \operatorname{diag}\left(\boldsymbol{V}^{*}\right) \operatorname{diag}\left(\boldsymbol{V}_{m}\right)^{-1}\right)\right) \\
& +2 \operatorname{diag}\left(\boldsymbol{B}_{c}^{*} / 2\right) \operatorname{abs}\left(\boldsymbol{M}_{b l}^{\mathrm{T}}\right) \operatorname{diag}\left(\boldsymbol{V}_{m}\right) \operatorname{diag}(\boldsymbol{\lambda})
\end{aligned}
$$

$\frac{\partial^{2}\left(\Delta \boldsymbol{S}^{\mathrm{T}} \boldsymbol{\lambda}\right)}{\partial \boldsymbol{z} \partial \boldsymbol{\theta}}=2 \operatorname{diag}\left(\boldsymbol{y}^{*}\right) \operatorname{imag}\left(\boldsymbol{M}_{S z v} \operatorname{diag}(\boldsymbol{\lambda}) \operatorname{diag}\left(\boldsymbol{V}^{*}\right)\right)$

$\boldsymbol{M}_{S z v}=\left[\begin{array}{c}\boldsymbol{V}^{\mathrm{T}} \boldsymbol{M}_{b l}(:, 1) \boldsymbol{M}_{b l}^{\mathrm{T}}(:, 1) \\ \vdots \\ \boldsymbol{V}^{\mathrm{T}} \boldsymbol{M}_{b l}\left(:, n_{l}\right) \boldsymbol{M}_{b l}^{\mathrm{T}}\left(:, n_{l}\right)\end{array}\right]$

where $\operatorname{real}(\cdot)$ and $\operatorname{imag}(\cdot)$ represent the real and the imaginary parts of the matrices, respectively; $\boldsymbol{M}_{S z v}$ is an $n_{l} \times n_{b}$ matrix, the $i$ th row of which is the $1 \times n_{b}$ vector $\boldsymbol{V}^{\mathrm{T}} \boldsymbol{M}_{b l}(:, i) \boldsymbol{M}_{b l}^{\mathrm{T}}(:, i)$.

The matrices derived above are necessary for the calculation process of the primal-dual interior point method to optimize the ACOPF problem with topological optimization.

\subsection{Islanding prevention}

Switching out transmission lines may lead to isolated buses. Therefore, islanding prevention measure is necessary for the optimization procedure.

First, on the basis of the bus-to-line incidence matrix, the buses that are connected by only one line are identified, and the lines connecting these buses cannot be switched out.

As for the islanding caused by multiple switching lines, the islanding prevention method is implemented as follows:

Step 1: Establish the bus-to-line incidence matrix on the basis of the original network and the set of the switched-out lines.

Step 2: From Bus 1, search the buses connected with Bus 1. For further search, find out the buses connected to the buses which are connected with Bus 1. Expand the search range gradually, and then determine the bus set $\Theta$ connected with Bus 1 directly or indirectly.

Step 3: Check whether $\Theta$ includes all the buses in the network. If yes, islanding has not been created; otherwise, islanding exists, and the switching decision is infeasible. Add the constraint as (33) that lines in the set cannot be switched out at the same time to the master problem.

\subsection{Complete procedure of ACOTS problem}

The complete procedure to solve the ACOTS problem is a two-level iterative optimization approach. The block diagram of the procedure is presented in Fig 1.

After the algorithm finds a feasible solution or doesn't converge, the solving process can be executed again by excluding the TS results found in the preceding iterations. The complete procedure can be run a number of times, and multiple solutions can be found and compared.

\section{Simulations and analysis}

The proposed method is tested on the modified IEEE 39-bus and IEEE 57-bus systems to identify the optimal line switching scheme for solving the voltage violation problem. The proposed method was implemented on a personal computer with $2.6 \mathrm{GHz}, 16 \mathrm{~GB}$ random access memory (RAM). Moreover, the solving time highly depends on system conditions and the initial values given for the optimization problem.

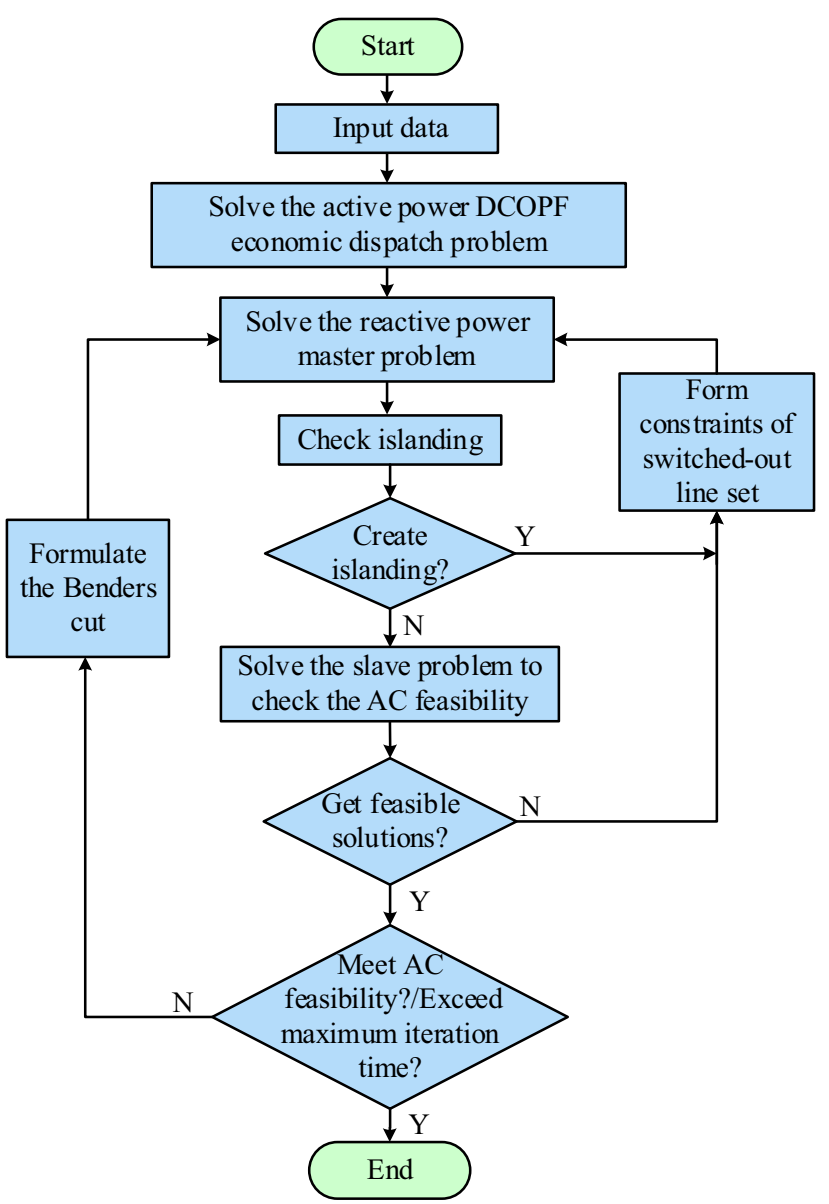

Fig. 1 Flowchart of the proposed method to solve ACOTS problem 


\subsection{IEEE 39-bus test system}

The IEEE 39-bus test system consists of 10 generators and 46 transmission lines. To make the power system operate in light-load condition with voltage violations, the total power load of the system is reduced by half to 3108.2 MW + j747.3 Mvar. In addition, the charging susceptance of each transmission line is doubled. In regard to the optimization parameters, in the slave problem, the voltage magnitude limits of the PQ buses are set as $V_{\mathrm{PQ}, i}^{\min }=0.96$ p.u., $V_{\mathrm{PQ}, i}^{\max }=1.04$ p.u..

In the linearized master problem, to ensure a suitable amount of voltage violation of the objective function, the voltage magnitudes of the PV buses are changed from 1.01 to 1.04 p.u., and their voltage magnitude limits are set a bit tighter than the limits of the slave problem, i.e., $V_{i}^{\min }=0.97$ p.u., $V_{i}^{\max }=1.03$ p.u..

The parameter of the voltage magnitude adjustment from the result of the master problem to the slave problem is set as $\gamma=2 \%$. The parameter of the generator active power adjustment to the slave problem is set as $\varepsilon=10 \%$. The maximum number of lines to be switched out is set as $N_{S}=3$. The convergence criterion is obtained by checking the objective value of the slave problem, which is set as $\eta \leq 0.001$.

The slave problem is first run to minimize the voltage violation quantity of the system without switching out lines. The objective function of the solution is $\eta=0.071$. The voltage magnitudes of the seven PQ buses violate the upper limits, whereas no lower limit is violated. The voltage magnitudes of the seven buses are presented in Table 1. The complete procedure to solve the ACOTS problem is then run for the IEEE 39-bus test system. The master problem is solved 28 times, which gives a line switching decision each time. Among them, only six topology results do not cause islanding, which are presented in Table 2. The total computation time is $194.7 \mathrm{~s}$.

For the six feasible topologies, the slave problems are solved, and Benders cuts are added to the master problem. The results of the slave problem are presented in the last column of Table 2. It can be seen that the objective function value of the master problem increased, while the value of the slave problem decreased monotonously until it became smaller than the convergence criterion 0.001, which is presented in Fig. 2.

In regard to the final result, lines 16,24 and 43 are switched out. The TS topology is illustrated in Fig. 3, in which the red lightning symbols denote the switched-out lines. It can be seen from the figure that the topology result may not meet the $N-1$ security criteria. For example, removing the line between Bus 26 and Bus 29 may cause islanding. In a practical operation, the switched-out lines
Table 1 Voltage magnitude violations of the 7 buses without TS

\begin{tabular}{lll}
\hline Bus number & Voltage magnitude (p.u.) & Violation quantity (p.u.) \\
\hline 10 & 1.053 & 0.013 \\
13 & 1.043 & 0.003 \\
14 & 1.052 & 0.012 \\
19 & 1.043 & 0.003 \\
26 & 1.063 & 0.023 \\
27 & 1.049 & 0.009 \\
28 & 1.048 & 0.008 \\
\hline
\end{tabular}

Table 2 Results of the 28 optimizations of master problem

\begin{tabular}{|c|c|c|c|c|}
\hline $\begin{array}{l}\text { Master } \\
\text { problem } \\
\text { iteration } \\
\text { time }\end{array}$ & $\begin{array}{l}\text { Objective } \\
\text { function value } \\
\text { of master } \\
\text { problem (p.u.) }\end{array}$ & $\begin{array}{l}\text { Switched- } \\
\text { out line }\end{array}$ & Islanding? & $\begin{array}{l}\text { Objective } \\
\text { function value } \\
\text { of slave } \\
\text { problem (p.u.) }\end{array}$ \\
\hline 1 & 0.032 & $42,43,44$ & Yes & \\
\hline 2 & 0.037 & $31,43,44$ & Yes & \\
\hline 3 & 0.047 & $1,43,44$ & Yes & \\
\hline 4 & 0.048 & $24,43,44$ & Yes & \\
\hline 5 & 0.053 & $30,43,44$ & Yes & \\
\hline 6 & 0.053 & $1,42,44$ & No & 0.0227 \\
\hline 7 & 0.056 & $16,43,44$ & Yes & \\
\hline 8 & 0.061 & $4,43,44$ & Yes & \\
\hline 9 & 0.061 & $9,43,44$ & Yes & \\
\hline 10 & 0.061 & $7,43,44$ & Yes & \\
\hline 11 & 0.063 & $36,43,44$ & Yes & \\
\hline 12 & 0.064 & $11,43,44$ & Yes & \\
\hline 13 & 0.065 & $13,43,44$ & Yes & \\
\hline 14 & 0.066 & $12,43,44$ & Yes & \\
\hline 15 & 0.066 & $25,43,44$ & Yes & \\
\hline 16 & 0.068 & $15,43,44$ & Yes & \\
\hline 17 & 0.068 & $40,43,44$ & Yes & \\
\hline 18 & 0.068 & $18,43,44$ & Yes & \\
\hline 19 & 0.069 & $29,43,44$ & Yes & \\
\hline 20 & 0.070 & $21,43,44$ & Yes & \\
\hline 21 & 0.070 & $8,43,44$ & Yes & \\
\hline 22 & 0.070 & 43,44 & Yes & \\
\hline 23 & 0.073 & $1,24,43$ & No & 0.0115 \\
\hline 24 & 0.074 & $1,24,44$ & No & 0.0106 \\
\hline 25 & 0.079 & $24,30,43$ & No & 0.0042 \\
\hline 26 & 0.082 & $1,16,44$ & Yes & \\
\hline 27 & 0.083 & $1,30,44$ & No & 0.0021 \\
\hline 28 & 0.085 & $16,24,43$ & No & 0.0000 \\
\hline
\end{tabular}

can be switched back timely to cope with the $N-1$ contingencies. The voltage magnitudes and active power 


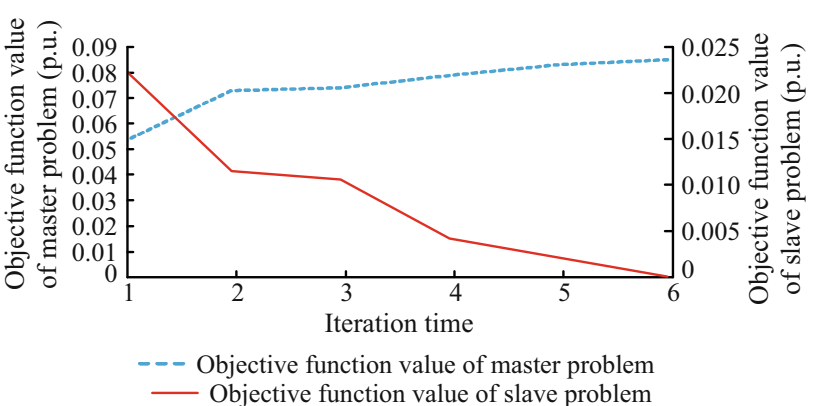

Fig. 2 Objective function values of master and slave problems of iterations

generation of the generators are also optimized, which are listed in Table 3. The voltage violation problem is solved.

\subsection{IEEE 57-bus test system}

The IEEE 57-bus test system consists of 7 generators and 80 transmission lines. To simulate the voltage violation conditions, the load power of the test system is reduced by half to a total load of $625.4 \mathrm{MW}+\mathrm{j} 168.2$ Mvar. The charging susceptances of the transmission lines are relatively low in the system, and therefore they are tripled for the simulation.

In regard to the optimization parameters, in the slave problem, voltage magnitude limits of the PQ buses of the slave problem are set as $V_{\mathrm{PQ}, i}^{\min }=0.95$ p.u., $V_{\mathrm{PQ}, i}^{\max }=1.05$ p.u.. In the linearized master problem, the voltage magnitudes of the PV buses are allowed to vary from 0.98 p.u. to 1.05 p.u., and the voltage magnitude limits of the buses are set as $V_{i}^{\min }=0.96$ p.u., $V_{i}^{\max }=1.04$ p.u..

The parameters of the voltage magnitude adjustment and generator active power adjustment are set the same as those for the IEEE 39-bus test system. The convergence criterion is also set to be the same as the former simulation, which is

Table 3 Optimization results of generators of IEEE 39-bus system

\begin{tabular}{llll}
\hline $\begin{array}{l}\text { Generator } \\
\text { number }\end{array}$ & $\begin{array}{l}\text { Bus } \\
\text { number }\end{array}$ & $\begin{array}{l}\text { Active power } \\
\text { generation (p.u.) }\end{array}$ & $\begin{array}{l}\text { Voltage } \\
\text { magnitude (p.u.) }\end{array}$ \\
\hline 1 & 30 & 1.157 & 0.9898 \\
2 & 31 & 3.158 & 0.9898 \\
3 & 32 & 3.284 & 0.9898 \\
4 & 33 & 3.262 & 0.9908 \\
5 & 34 & 2.245 & 0.9898 \\
6 & 35 & 3.536 & 0.9898 \\
7 & 36 & 2.633 & 0.9898 \\
8 & 37 & 2.845 & 0.9898 \\
9 & 38 & 4.103 & 0.9898 \\
10 & 39 & 4.994 & 1.0001 \\
\hline
\end{tabular}

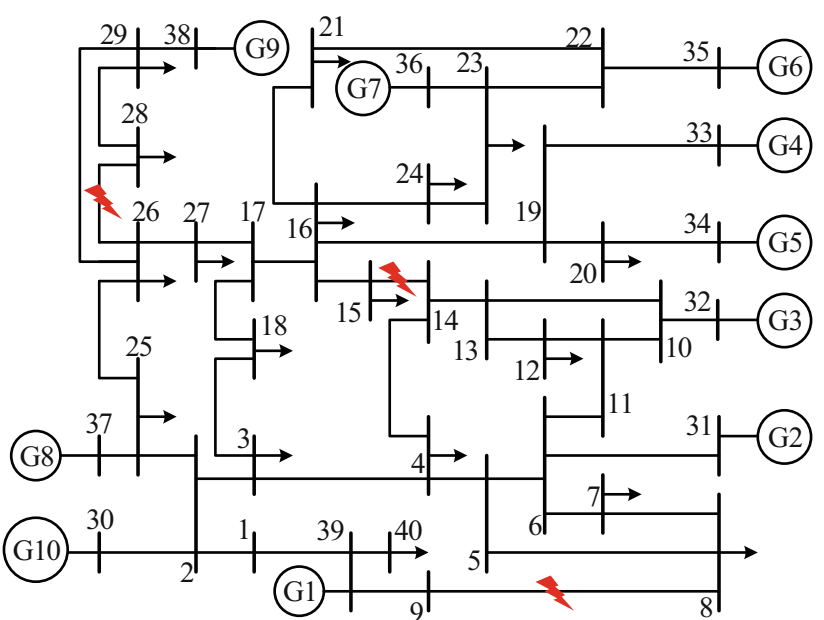

Fig. 3 Topology illustration of IEEE 39-bus system after TS

$\eta \leq 0.001$. The maximum number of lines that can be switched out is set as $N_{S}=5$. The slave problem is run first to minimize the voltage violation quantity of the system without switching out lines. The objective function of the solution is $\eta=0.1447$.

The voltage magnitudes of the 16 PQ buses violate the upper limits, while no lower limit is violated. The voltage magnitudes of the 16 buses are presented in Table 4 .

The complete procedure is then run, and the master problem is solved 10 times. Because this test system is quite strong, the network topologies are all feasible for the 10 iterations. The results of the 10 iterations are presented in Table 5. The total computation time is $324.7 \mathrm{~s}$.

From Table 5, it can be seen that the objective function value of the master problem increases, while the objective

Table 4 Voltage magnitude violations of the 16 buses without TS

\begin{tabular}{lll}
\hline Bus number & Voltage magnitude (p.u.) & Violation quantity (p.u.) \\
\hline 21 & 1.0504 & 0.0004 \\
22 & 1.0516 & 0.0016 \\
23 & 1.0517 & 0.0017 \\
24 & 1.0541 & 0.0041 \\
25 & 1.0791 & 0.0291 \\
30 & 1.0720 & 0.0220 \\
31 & 1.0606 & 0.0106 \\
32 & 1.0575 & 0.0075 \\
33 & 1.0569 & 0.0069 \\
38 & 1.0515 & 0.0015 \\
46 & 1.0726 & 0.0226 \\
47 & 1.0615 & 0.0115 \\
48 & 1.0588 & 0.0088 \\
49 & 1.0620 & 0.0120 \\
50 & 1.0527 & 0.0027 \\
51 & 1.0517 & 0.0017 \\
\hline
\end{tabular}


Table 5 Results of 10 iterations of the decomposition approach

\begin{tabular}{llll}
\hline Iteration time & Switched-out line & $\begin{array}{l}\text { Objective function value } \\
\text { of master problem (p.u.) }\end{array}$ & $\begin{array}{l}\text { Objective function value } \\
\text { of slave problem (p.u.) }\end{array}$ \\
\hline 1 & $21,31,52,63,76$ & 0.0036 & 0.4247 \\
2 & $21,31,51,63,73$ & 0.0036 & 0.2728 \\
3 & $21,32,52,63,76$ & 0.0036 & 0.1051 \\
4 & $4,31,51,63,73$ & 0.0036 & 0.0316 \\
5 & $21,32,63,73,76$ & 0.0036 & 0.0259 \\
6 & $18,30,51,63,70$ & 0.0041 & 0.0192 \\
7 & $18,30,63,73,76$ & 0.0041 & 0.0167 \\
9 & $12,21,51,63,73$ & 0.0042 & 0.0251 \\
10 & $18,25,30,52,63$ & 0.0057 & 0.0076 \\
\end{tabular}

Table 6 Optimization results of generators of IEEE 57-bus system

\begin{tabular}{llll}
\hline $\begin{array}{l}\text { Generator } \\
\text { number }\end{array}$ & $\begin{array}{l}\text { Bus } \\
\text { number }\end{array}$ & $\begin{array}{l}\text { Active power } \\
\text { generation (p.u.) }\end{array}$ & $\begin{array}{l}\text { Voltage } \\
\text { magnitude (p.u.) }\end{array}$ \\
\hline 1 & 1 & 210.84 & 1.003964 \\
2 & 2 & 27.04 & 0.993841 \\
3 & 3 & 22.15 & 0.988985 \\
4 & 6 & 14.18 & 0.991363 \\
5 & 8 & 214.14 & 0.988530 \\
6 & 9 & 22.60 & 0.983563 \\
7 & 12 & 138.63 & 0.979223 \\
\hline
\end{tabular}

function value of the slave problem decreases monotonously. For the last iteration, the lines with numbers 12 , $15,18,32$, and 63 are switched out. The voltage magnitudes and active power generation of the generators are also optimized, and are presented in Table 6 . The voltage violations are all eliminated.

In consideration of the discussion in Sect. 3.2, the global optimal solution may be removed by the Benders cut formulated by the non-convex slave problem, and as a result the final solution may be just a local optimal solution. To obtain better solutions, the complete solution procedure presented in Sect. 3.5 can be executed iteratively by excluding the TS solutions found in the preceding iterations. Different sets of TS results can then be compared and selected.

After the first procedure converges, an optimal set of switching lines have been chosen. To get other feasible solutions, an additional constraint as (33) that the lines in the former set cannot be switched out at the same time is added. The procedure is run again with seven iterations, and the results are presented in Table 7. The total time for finding the second solution is $211.6 \mathrm{~s}$. By switching out the lines with numbers $5,21,25,63$, and 73 , voltage violations are eliminated.

Repeat the above procedure and solve the problem for the third time. In this time, no feasible solutions can be obtained. Therefore, the two sets of switched-out lines with numbers $5,21,25,63,73$ and 12, 15, 18, 32, 63 are both feasible TS results to eliminate voltage violations for this simulation case. The feasible TS results can be further compared from different perspectives, such as the switched-out costs of different transmission lines.

Table 7 Results of 7 iterations of the second procedure

\begin{tabular}{llll}
\hline Iteration time & Switched-out line & $\begin{array}{l}\text { Objective function value } \\
\text { of master problem (p.u.) }\end{array}$ & $\begin{array}{l}\text { Objective function value } \\
\text { of slave problem (p.u.) }\end{array}$ \\
\hline 1 & $4,32,52,63,76$ & 0.0036 & 0.1011 \\
2 & $21,32,63,73,76$ & 0.0036 & 0.0259 \\
3 & $18,30,63,73,76$ & 0.0041 & 0.0167 \\
4 & $8,21,52,63,76$ & 0.0042 & 0.0954 \\
5 & $5,21,51,63,76$ & 0.0042 & 0.0090 \\
7 & $5,18,21,32,63$ & 0.0057 & 0.0480 \\
\hline
\end{tabular}




\section{Conclusion}

In this paper, TS is employed to eliminate voltage violations for transmission systems in lightly loaded conditions. The TS problem with AC power flow constraints is proposed as an MINLP problem. To solve this problem, the decomposition approach is used to decompose the original problem into a linearized master problem and an ACOPF slave problem. The master problem optimizes the switching scheme and voltage magnitudes under linearized constraints, and the slave problem is used to check the AC feasibility. To apply the interior point method for solving the slave problem, Jacobian and Hessian matrices of the AC power flow equations considering topological change are derived. In addition, a measure to prevent islanding is proposed. Simulation results on two test systems demonstrate the effectiveness of the proposed method.

From the simulation results, it can be seen that the proposed decomposition approach gives satisfactory convergence performance. Although the decomposition approach cannot guarantee global optimality, the complete procedure can be run several times to get different sets of feasible solutions which are acceptable for the purpose of eliminating voltage violations. The relatively optimal solutions can be determined by comparison from diverse perspectives. Future work will focus on the optimization method considering different load levels and operational conditions.

Open Access This article is distributed under the terms of the Creative Commons Attribution 4.0 International License (http:// creativecommons.org/licenses/by/4.0/), which permits unrestricted use, distribution, and reproduction in any medium, provided you give appropriate credit to the original author(s) and the source, provide a link to the Creative Commons license, and indicate if changes were made.

\section{References}

[1] Khodaei A, Shahidehpour M (2010) Transmission switching in security-constrained unit commitment. IEEE Trans Power Syst 25(4):1937-1945

[2] Hedman KW, Ferris MC, O'Neill RP et al (2010) Co-optimization of generation unit commitment and transmission switching with $n-1$ reliability. IEEE Trans Power Syst 25(2):1052-1063

[3] Khanabadi M, Ghasemi H, Doostizadeh M (2013) Optimal transmission switching considering voltage security and $n-1$ contingency analysis. IEEE Trans Power Syst 25(2):542-550

[4] Ostrowski J, Wang J, Liu C (2014) Transmission switching with connectivity-ensuring constraints. IEEE Trans Power Syst 29(6):2621-2627

[5] Heidarifar M, Ghasemi H (2016) A network topology optimization model based on substation and node-breaker modeling. IEEE Trans Power Syst 31(1):247-255
[6] Han J, Papavasiliou A (2015) Congestion management through topological corrections: a case study of central western Europe. Energy Policy 86:470-482

[7] Escobedo AR, Moreno-Centeno E, Hedman KW (2014) Topology control for load shed recovery. IEEE Trans Power Syst 29(2):908-916

[8] Liu WL, Chiang HD (2015) Toward on-line line switching method for relieving overloads in power systems. In: Proceedings of 2015 IEEE power and energy society general meeting, Denver, USA, 26-30 July 2015, 5 pp

[9] Yang Z, Zhong H, Xia Q et al (2016) Optimal transmission switching with short-circuit current limitation constraints. IEEE Trans Power Syst 31(2):1278-1288

[10] Hedman KW, Oren SS, O'Neill RP (2011) A review of transmission switching and network topology optimization. In: Proceedings of 2011 IEEE power and energy society general meeting, San Diego, USA, 24-29 July 2011, 7 pp

[11] Bacher R, Glavitsch H (1988) Loss reduction by network switching. IEEE Trans Power Syst 3(2):447-454

[12] Quintana VH (1990) Overload and voltage control of power systems by line switching and generation rescheduling. Can J Electr Comput Eng 15(4):167-173

[13] Fliscounakis S, Zaoui F, Simeant G et al (2007) Topology influence on loss reduction as a mixed integer linear programming problem. In: Proceedings of IEEE Lausanne PowerTech, Lausanne, Switzerland, 1-5 July 2007, pp 1987-1990

[14] Shao W, Vittal V (2005) Corrective switching algorithm for relieving overloads and voltage violations. IEEE Trans Power Syst 20(4):1877-1885

[15] Guo WM, Wei Q, Liu GJ et al (2013) Transmission switching to relieve voltage violations in low load period. In: Proceedings of 4th IEEE PES innovative smart grid technologies, Lyngby, Denmark, 6-9 October 2013, 5 pp

[16] Bienstock D, Munoz G (2015) Approximate method for AC transmission switching based on a simple relaxation for ACOPF problems. In: Proceedings of IEEE PES general meeting, Denver, USA, 26-30 July 2015, 5 pp

[17] Bai Y, Zhong H, Xia Q et al (2015) A conic programming approach to optimal transmission switching considering reactive power and voltage security. In: Proceedings of IEEE PES general meeting, Denver, USA, 26-30 July 2015, 5 pp

[18] Bai Y, Zhong H, Xia Q et al (2017) A two-level approach to AC optimal transmission switching with an accelerating technique. IEEE Trans Power Syst 32(2):1616-1625

[19] Soroush M, Fuller JD (2014) Accuracies of optimal transmission switching heuristics based on DCOPF and ACOPF. IEEE Trans Power Syst 29(2):924-932

[20] Coffrin C, Hijazi H, Lehmann K et al (2014) Primal and dual bounds for optimal transmission switching. In: Proceedings of the 18th power systems computation conference, Wroclaw, Poland, 18-22 August 2014, 8 pp

[21] Lotfjou A, Shahidehpour M, Fu Y et al (2010) Security-constrained unit commitment with AC/DC transmission systems. IEEE Trans Power Syst 25(1):531-542

[22] Wang B, Xia Y, Xia Q et al (2016) Security-constrained economic dispatch with AC/DC interconnection system based on Benders decomposition method. Proc CSEE 36(6):1-8

[23] Huang S, Dinavahi V (2016) Security constrained transmission expansion planning by accelerated Benders decomposition. In: Proceedings of north American power symposium (NAPS), Denver, USA, 18-20 September 2016, 6 pp

[24] Ajaja A, Galiana FD (2013) Optimal reconfiguration of distribution networks using MILP and supporting hyperplanes (HYPER). In: Proceedings of IEEE power energy society general meeting, Vancouver, Canada, 21-25 July 2013, 5 pp 
[25] Mínguez R, Milano F, Zárate-Miñano R et al (2007) Optimal network placement of SVC devices. IEEE Trans Power Syst 22(4):1851-1860

[26] Li C, Guo Z, Fan A (2002) Application of primal-dual interior point method of optimal power flow to power system. Electr Power Autom Equip 22(8):4-7

[27] Chen Q, Guo R (2008) A hybrid reactive power optimization algorithm based on improved genetic algorithm and primal-dual interior point algorithm. Power Syst Technol 32(24):50-54

[28] Zimmerman RD, Murillo-Sánchez CE, Thomas RJ (2011) MATPOWER: steady-state operations, planning and analysis tools for power systems research and education. IEEE Trans Power Syst 26(1):12-19

Boshi ZHAO received the B.S. degree in electrical engineering from Tsinghua University, China, in 2013, where he is still continuing in Ph.D. course. His research interests include operation and planning of electrical transmission systems, voltage stability and electricity market.

Zechun HU received the B.S. and Ph.D. degrees in electrical engineering from Xi' an Jiaotong University, China, in 2000 and 2006, respectively. He was a former lecturer and associate professor in Shanghai Jiao Tong University, China, and he was a research officer in University of Bath, UK. He is currently working as an associate professor and doctoral supervisor in the Department of Electrical Engineering, Tsinghua University, China. His research interests include operation and optimization of power systems, interaction of electric vehicles and power systems, demand side response, and electricity market.
Qian ZHOU received the B.S. and M.S. degrees in electrical engineering from Xi' an Jiaotong University, China, in 2001 and 2005, respectively. He is working as a senior engineering in Jiangsu Electric Power Company Research Institute of State Grid Corporation of China. His research interests include reactive power planning of power system, voltage stability and analysis and control of safe and stable operation of power systems.

Hongcai ZHANG received the B.S. and Ph.D. degrees in electrical engineering from Tsinghua University, China, in 2013 and 2018, respectively. He is holding a postdoctoral position in University of California Berkeley, US. His research interests include operation and optimization of power systems, interaction of electric vehicles and power systems.

Yonghua SONG received the B.S. degree in electrical engineering from University of Science and Technology of Chengdu, China, in 1984 and the Ph.D. degree from China Electric Power Research Institute in 1989. He was a former professor and vice president of University of Brunel and University of Liverpool, UK. He was also the former deputy president of Zhejiang University, China. He is currently working as a professor in Tsinghua University and the president of University of Macau, Macau, China. His research interests include stability analysis of power system, operation and optimization of power systems and electric vehicles. 